\title{
Invasive Klebsiella pneumoniae Infections in Community-Settings and Healthcare Settings
}

\author{
Yue Qiu, ${ }^{1, *}$ Daojiong Lin, ${ }^{2, *}$ Yi Xu, ${ }^{3, *}$ \\ Yibing Cheng, ${ }^{4}$ Fang Wang, ${ }^{5}$ \\ Qingxiong Zhu, ${ }^{6}$ Chunhui Zhu, ${ }^{6}$ \\ Chaomin Wan, ${ }^{7}$ Yu Zhu, ${ }^{7}$ \\ Jianning Tong, ${ }^{8}$ Rui Li, ${ }^{8}$ \\ Qionghua Zhou, ${ }^{2}$ Minxia Chen, (D) $^{3}$ \\ Qingwen Shan, ${ }^{9}$ Zhiqiang Zhuo, ${ }^{10}$ \\ Caihong Wang, ${ }^{10}$ Shiyong Zhao, " \\ Wen Song, "' Mei Zeng'
}

'Department of Infectious Diseases, Children's Hospital of Fudan University, ShangHai, People's

Republic of China; ${ }^{2}$ Department of Infectious

Diseases, Hainan Women and Children's

Medical Center, Haikou, People's Republic of

China; ${ }^{3}$ Department of Infections Disease,

Guangzhou Women and Children's Medical

Center, Guangzhou, People's Republic of China;

${ }^{4}$ Department of Emergency, Children's Hospital

Affiliated to Zhengzhou University (Henan

Children's Hospital), Zhengzhou, People's

Republic of China; ${ }^{5}$ Department of Infections

Disease, Children's Hospital Affiliated to

Zhengzhou University (Henan Children's

Hospital), Zhengzhou, People's Republic of

China; ${ }^{6}$ Department of Infection Diseases,

Children's hospital of Jiangxi Province,

Nanchang, People's Republic of China;

${ }^{7}$ Department of Pediatrics, West China Second

Hospital, Sichuan University, Chengdu, People's

Republic of China; ${ }^{8}$ Department of Pediatric,

Gastroenterology and Infectious Diseases,

Qingdao Women and Children's Hospital,

Qingdao, People's Republic of China;

${ }^{9}$ Department of Pediatrics, The First Affiliated

Hospital of Guangxi Medical University,

Nanning, People's Republic of China;

${ }^{10}$ Department of Infectious Diseases, Xiamen

Children's Hospital, Xiamen, People's Republic

of China; "Department of Infectious Diseases,

Hangzhou Children's Hospital, Hangzhou,

People's Republic of China

*These authors contributed equally to this work

Correspondence: Mei Zeng

Department of Infectious Diseases,

Children's Hospital of Fudan University,

399 Wanyuan Road, Shanghai, 201102,

People's Republic of China

Tel +86-2I-6493 I I 32

Email zengmeigao@aliyun.com
Objective: To assess clinical characteristics, outcomes and antimicrobial resistance of invasive Klebsiella pneumoniae (KP) infections in Chinese pediatric patients in hospital and community settings.

Methods: This retrospective study was conducted in the nine tertiary hospitals during 20162018. The 324 pediatric inpatients who had KP isolated from blood and cerebrospinal fluid and had complete medical records reviewed were included. We analyzed the risk factors, outcomes and antimicrobial resistance pattern of KP-infected patients based on comparison between healthcare-associated KP infections (HAI) and community-acquired infections.

Results: Of the 324 enrolled patients, 275 (84.9\%) were clinically defined as HAI, including $175(63.6 \%)$ neonates and $100(36.4 \%)$ aged $>28$ days. The overall prevalence of CRKP was $38.2 \%$ (43.4\% in HAI verse $8.7 \%$ in CAI, $\mathrm{P}<0.05$ ). Prematurity (odds ratio (OR): 37.07 , 95\% CI: 8.29-165.84), hematologic malignancies (OR: 15.52, 95\% CI: 1.89-127.14) and invasive mechanical ventilation (OR: 13.09 , 95\% CI: 1.66-103.56) were independent risk factors for HAI. Patients from rural area (OR: 1.94, 95\% CI: 1.12-3.35), invasive mechanical ventilation (OR: $2.33,95 \%$ CI: $1.25-4.33$ ), antibiotic therapy prior to admission (OR: 2.33 , 95\% CI: $1.25-4.33$ ) and prior hospital stay in the past 30 days (OR: 3.46, 95\% CI: 1.87 6.41) were associated with healthcare-associated CRKP infections. Organ dysfunction was independently correlated with poor outcomes (OR: 2.92, 95\% CI: 1.23-6.95).

Conclusion: Pediatric invasive KP infections and high prevalence of CRKP infections largely occurred in healthcare settings in China. The adequate and intensified infection control measures should be focused on high-risk hematologic patients, neonatal patients and intubated patients.

Keywords: Klebsiella pneumoniae, healthcare-associated infections, children

\section{Introduction}

Klebsiella pneumoniae (KP) has been implicated in severe infections and one of the most important causes of nosocomial infections. ${ }^{1}$ According to the global surveillance data, KP is the third leading bloodstream pathogen in children, and the frequency of antibiotic resistant KP has been increasing over years. ${ }^{2-4}$ In recent years. Carbapenem-resistant Klebsiella pneumoniae (CRKP) represents a "critical concern" of the World Health Organization and poses great challenges for clinical treatment. ${ }^{5,6}$ Of particular concern, the prevalence of CRKP has been sharply rising from $2.9-3 \%$ in 2005 to $25-26.3 \%$ in 2018 in China, and even reached $35.8 \%$ in neonatal patients. ${ }^{4,7}$

Currently, KP infections become an important clinical issue in pediatrics, especially in neonates, since very limited effective antibiotics are available for the 
treatment choice. ${ }^{8,9}$ The existing studies from other countries showed KP as a key opportunistic pathogen causing severe infections in hospitalized children. ${ }^{10-13}$ So far, surveillance study and clinical data on healthcare-associated $\mathrm{KP}$ infections are lacking in China, although CRKP is now a pronounced problem. According to some regional surveillance studies, most of Carbapenem-resistant organisms (CRO) are healthcare-associated. ${ }^{10,14}$ Therefore, we carried out this retrospective multicenter study to understand the current situations of invasive healthcare-associated KP infections in Chinese pediatric patients, and analyzed risk factors, outcomes and antimicrobial resistance of healthcare-associated KP and CRKP infections, aiming to identify the main problem of invasive KP infections and help find the effective solution to reduce and prevent healthcare-associated KP and CRKP infections in pediatric patients.

\section{Method}

\section{Study Design and Subjects}

This is a retrospective multicenter study conducted between January 2016 and December 2018 at the nine tertiary hospitals in China, including four provincial Children's Hospitals, two General Hospitals and three municipal Women and Children's Hospitals. These hospitals locate in different administrative regions of China: Five hospitals in the Southeast, three in the South and one in Central, respectively. The inclusion criteria of eligible patients were as follows: 1) hospitalized pediatric inpatients aged $<18$ years, 2 ) inpatients who had KP isolated from blood and/or cerebrospinal fluid (CSF) specimens. The patients were excluded if their clinical data collection was incomplete or their medical records were unavailable for review by the end of 2019 .

\section{Definitions}

Healthcare-associated infections (HAI) were defined as follows: 1) KP strain recovered from sterile specimens collected at least $48 \mathrm{~h}$ after hospital admission in a patient; 2) KP culture positive within the first 48 hours of admission in a patient with prior hospital stay for a few of consecutive days in the past 30 days. Communityacquired infections (CAI) were defined as KP culture positive within 48 hours of admission in a patient without prior hospitalization within the past 30 days. Poor outcomes were considered if a patient died in hospital or was hopelessly discharged from hospital under the request of parents based on the medical records.

\section{Data Collection}

The following data were collected, including patient's demographics and important underlying diseases, previous history of antibiotic use and hospitalization within the 30 days prior to the admission date, ward unit during hospitalization, clinical diagnosis on admission and discharge, length of hospitalization, interval between the occurrence of KP infections (the collection date of the first KPpositive sample) and the date of admission, intravascular catheter use, mechanical ventilation, imaging findings, clinical outcomes and microbiological data.

\section{Microbiological Study}

The first invasive KP isolate recovered from a patient was included in this study for microbiological analysis. Species identification and antimicrobial susceptibility testing were performed at local hospital laboratories by automated systems such as Vitek or Phoenix, according to the 2018 CLSI performance guideline. ${ }^{15}$ Antimicrobial susceptibility testing was performed for clinical isolates using minimum inhibitory concentrations (MICs, microdilution method-based) or Kirby-Bauer disk diffusion method. The original results were classified according to the breakpoints of the 2018 CLSI standards. For polymyxin B, the criteria referred to the epidemiological cutoff value of colistin in the CLSI file (MIC $\leq 2 \mu \mathrm{g} / \mathrm{mL}$ for wild strains; $\mathrm{MIC} \geq 4 \mu \mathrm{g} / \mathrm{mL}$ for non-wild strains). ${ }^{7}$

\section{Statistical Analysis}

Data were entered and analyzed into Excel version 2016 (Microsoft, Redmond, Washington) and were analyzed using SPSS (IBM Statistics 20.0). Results were described as absolute numbers and percentage or as median and interquartile ranges. Categorical variables were compared using the Chi-square or Fisher's exact tests. Continuous variables were compared by Student's $t$-test or MannWhitney $U$-test according to their distribution. The risk factors were analyzed by Binary regression analysis and Multinomial regression analysis, and the results were presented as odds ratios (ORs), 95\% confidence intervals (CIs) and P values. Significant variables with $\mathrm{P}$ value of $<0.2$ were then selected for multiple regression analysis to evaluate risk factors for clinical poor outcomes. A difference with $\mathrm{P}<0.05$ was considered to be statistically significant. 


\section{Result}

The Basic Characteristics of KP-Infected Patients

A total of 425 pediatric inpatients met the diagnosis criteria of invasive KP infections based on the hospital microbiology laboratory records, of whom, 248 (58.4\%) neonates $\leq 28$ days and $177(41.6 \%)$ were children aged $>28$ days on admission. Finally, 324 (76.2\%) patients met the enrollment criteria and were included in this study and $101(23.8 \%)$ were excluded due to unavailability of medical records (99 patients) and incomplete clinical data collection ( 2 patients).

Of the 324 enrolled patients, 299 (92.3\%) had KP isolated from blood, $11(3.4 \%)$ had $\mathrm{KP}$ isolated from CSF and 14 (4.3\%) had KP isolated from both blood and CSF. On the clinical grounds and case definition, 275 (84.9\%) patients were considered as HAI and 49 (15.1\%) patients were considered as CAI.

\section{Clinical Features and Risk Factors of Healthcare-Associated KP Infections in Pediatric Patients}

Among 275 patients with HAI, 154 (56\%) were male; the median age was 0.6 months (IQR 0.17-6, range: 1 day-17 years) and the age distribution was as followings: 162 $(58.9 \%)$ aged $\leq 28$ days, $33(12.0 \%)$ aged 29 days-2 months, 20 (7.3\%) aged 3-11 months, 33 (12.0\%) aged $1-5$ years, $27(9.8 \%)$ aged $>5$ years. Of 162 neonates, 129 $(79.6 \%)$ were preterm, including $13(10.1 \%)$ at $<28$ weeks of gestational age, $75(58.1 \%)$ at 28-31 weeks of gestational age and $41(31.8 \%)$ at $32-37$ weeks of gestational age. Two hundred and eighteen $(79.3 \%)$ patients had the accompanying focal organ infections, including pneumonia $(119,43.3 \%)$, meningitis $(64,23.3 \%)$, intra-abdominal infections $(41,14.9 \%)$, upper urinary tract infections (3, $1.1 \%$ ) and osteomyelitis $(4,1.5 \%) ; 13$ patients had 2 infection sites. Nine (3.3\%) patients died in hospital and $30(10.9 \%)$ patients were hopelessly discharged at the request of parents.

Compared to CAI patients (Table 1), the percentage of patients with underlying medical conditions was significantly greater in HAI patients, including hematologic malignancies, necrotizing enterocolitis (NEC) and prematurity $(16.4 \%$ versus $2 \%, 9.8 \%$ versus $0 \%, 46.9 \%$ versus $4.1 \%$, respectively; $\mathrm{P}<0.05)$; the percentage of patients using peripherally inserted central catheter (PICC), invasive mechanical ventilation was significantly greater in HAI patients $(29.8 \%$ versus $2 \%, 34.9 \%$ versus $2 \%$, respectively, $\mathrm{P}<0.01)$. HAI patients had more episodes of meningitis than CAI patients $(23.3 \%$ versus $6.1 \%$, $\mathrm{P}<0.01$ ), while CAI patients had more frequency of upper urinary tract infections than HAI patients $(30.6 \%$ versus $1.1 \%, \mathrm{P}<0.01)$. The isolation rate of CRKP strains was much higher in HAI patients than in CAI patients (43.4\% versus $8.7 \%, \mathrm{P}<0.01$ ). The poor outcomes were similar between HAI and CAI patients (14.2\% versus $8.2 \%, \mathrm{P}>0.05)$.

Multiple regression analysis showed that prematurity ((odds ratio) OR: 37.07, 95\% CI: 8.29-165.84), hematologic malignancies (OR: 15.52, 95\% CI: 1.89-127.14), and invasive mechanical ventilation (OR: 13.09, 95\% CI: 1.66-103.56) were independent risk factors for HAI (Table 2).

\section{Risk Factors of Healthcare-Associated CRKP Infections in Pediatric Patients}

Compared to CSKP-infected patients (Table 3), CRKPinfected patients stayed in hospital for a longer time (38.5 days versus 30 days, $\mathrm{P}<0.05$ ) and received more therapies of mechanical ventilation (45.5\% versus $26.7 \%$, $\mathrm{P}<0.05)$. More CRKP-infected patients developed organ dysfunction than CSKP-infected patients $(36.1 \%$ versus $15.1 \%, \mathrm{P}<0.05)$. The poor outcomes were similar between CSKP-infected and CRKP-infected patients (14.3\% versus $13 \%, \mathrm{P}>0.05)$.

Multiple regression analysis showed that children from rural area (OR: $1.94,95 \% \mathrm{CI}: 1.12-3.35, \mathrm{P}<0.05$ ), invasive mechanical ventilation (OR: $2.08,95 \%$ CI: 1.12-3.88, $\mathrm{P}<0.05$ ), prior antibiotic therapy before hospitalization (OR: $2.33,95 \%$ CI: $1.25-4.33, \mathrm{P}<0.01$ ) and prior hospitalization in the past 30 days (OR: $3.46,95 \%$ CI: $1.87-$ 6.41, P <0.01) were independent risk factors for CRKP infections (Table 4).

\section{Risk Factors of Poor Outcomes in Pediatric Patients with Healthcare-Associated KP Infections}

As shown in Table 5, univariate analysis showed that invasive mechanical ventilation, any organ dysfunction and septic shock were the significant risk factors for poor outcomes. Multiple regression analysis showed that organ dysfunction was an independent risk factor for the poor outcomes (OR: 2.92, [95\% CI: 1.23-6.95], P <0.05). 
Table I Characteristics of the Children with Invasive Klebsiella pneumoniae Infection According to Type of Acquired Infections (HAl or CAI)

\begin{tabular}{|c|c|c|c|c|}
\hline Variable & Total $(n=324)$ & HAI $(\mathbf{N}=275)$ & CAI $(N=49)$ & $P$ value \\
\hline Age (months) & $0.8(0.3-6.4)$ & $0.6(0.17-6)$ & $3.0(0.87-10)$ & 0.54 \\
\hline Male & $187(57.7)$ & $154(56)$ & $33(67.3)$ & 0.14 \\
\hline Rural area & $178(54.9)$ & $156(56.7)$ & $22(44.9)$ & 0.13 \\
\hline \multicolumn{5}{|l|}{ Underlying disease } \\
\hline Hematologic malignancies & $45(13.9)$ & $44(16)$ & $\mathrm{I}(2)$ & 0.02 \\
\hline Congenital anomalies & $42(13)$ & $37(13.5)$ & $5(10.2)$ & 0.53 \\
\hline NEC & $27(8.3)$ & $27(9.8)$ & $0(0)$ & 0.02 \\
\hline Prematurity & |3| (40.4) & $129(46.9)$ & $2(4.1)$ & 0 \\
\hline Solid tumors & $4(1.2)$ & $4(1.5)$ & $0(0)$ & 1 \\
\hline Severe Malnutrition & $12(3.7)$ & II (4) & I (2) & 0.8 \\
\hline Immunodeficiencies & $3(0.9)$ & $3(1.1)$ & $0(0)$ & 1 \\
\hline \multicolumn{5}{|l|}{ Departments } \\
\hline - Hematology-oncology ward & $42(13)$ & $42(15.3)$ & $0(0)$ & $<0.01$ \\
\hline - General medical ward & $157(48.5)$ & $119(43.3)$ & $38(77.6)$ & 0 \\
\hline$\bullet \mathrm{NICU}$ & $65(20.1)$ & $64(23.3)$ & I (2) & 0 \\
\hline - PICU & $21(6.5)$ & $18(6.5)$ & $3(6.1)$ & 1 \\
\hline - Surgical ward & $39(12)$ & $32(11.6)$ & $7(14.3)$ & 0.6 \\
\hline Length of hospitalization & $28(\mid 7-50)$ & $33(2 I-53.5)$ & $13(9-16)$ & 0 \\
\hline Length of hospitalization before the onset of KP infections & $7(1.8-20)$ & $10(2-22)$ & $I(I-I)$ & 0 \\
\hline \multicolumn{5}{|l|}{ Invasive procedures and devices } \\
\hline $\mathrm{PICC}$ & $83(25.6)$ & $82(29.8)$ & I (2) & 0 \\
\hline Thoracic cavity drainage & $12(3.7)$ & $12(4.4)$ & $0(0)$ & 0.23 \\
\hline Abdominal indwelling catheter & $4(1.2)$ & $4(1.5)$ & $0(0)$ & 1 \\
\hline Indwelling urinary catheter & $8(2.5)$ & $5(1.8)$ & $3(6.1)$ & 0.2 \\
\hline Invasive mechanical ventilation & $97(29.9)$ & $96(34.9)$ & $I(2)$ & 0 \\
\hline Surgical operation & $65(20.1)$ & $60(21.8)$ & $5(10.2)$ & 0.06 \\
\hline Organ dysfunction (any) & $73(22.5)$ & $69(25.1)$ & $4(8.2)$ & 0.015 \\
\hline Septic shock & $32(9.9)$ & $30(10.9)$ & $2(4.1)$ & 0.22 \\
\hline Mortality or hopeless discharge & $44(13.6)$ & $39(14.2)$ & $5(8.2)$ & 0.45 \\
\hline Isolation of CRKP $(\mathrm{N}=304)$ & $116(38.2)$ & $112(43.4)$ & $4(8.7)$ & 0 \\
\hline \multicolumn{5}{|l|}{ Combined organ infection } \\
\hline Pneumonia & $139(42.9)$ & $119(43.3)$ & $20(40.8)$ & 0.75 \\
\hline Meningitis & $67(20.1)$ & $64(23.3)$ & $3(6.1)$ & 0 \\
\hline Intrabdominal infections & $46(14.2)$ & $4 I(14.9)$ & $5(10.2)$ & 0.38 \\
\hline Upper urinary tract infections & $18(5.6)$ & $3(1.1)$ & $15(30.6)$ & 0 \\
\hline
\end{tabular}

Abbreviations: HAI, healthcare-associated infections; CAI, community-acquired infections; PICC, peripherally inserted central catheter; NEC, necrotizing enterocolitis; NICU, neonatal intensive care unit; PICU, pediatric intensive care unit; CRKP, carbapenem-resistant Klebsiella pneumoniae.

\section{Antimicrobial Susceptibility Patterns of Healthcare-Associated KP Isolates}

As shown in Table 6, healthcare-associated KP strains showed high frequency of resistance to carbapenem as well as other clinical important antibiotics usually recommended for the treatment of Enterobacteriaceae infections, such as thirdgeneration cephalosporins, cefepime and piperacillin/ tazobactam. Also, healthcare-associated KP strains showed significantly higher frequency of resistance to clinical important antibiotics than community-acquired KP stains. Besides, healthcare-associated KP strains showed relatively higher resistance percentage to tigecycline, amikacin, ciprofloxacin and levofloxacin (12.5-23.7\%). Healthcare-associated KP strains almost remained sensitive to polymyxin B. 
Table 2 Multiple Regression Analysis of Risk Factors Associated with HAI

\begin{tabular}{|c|c|c|c|c|c|c|}
\hline \multirow[t]{2}{*}{ Variable } & \multirow[t]{2}{*}{ Total } & \multirow[t]{2}{*}{ HAl } & \multirow[t]{2}{*}{ CAI } & \multicolumn{3}{|c|}{ Multi-Variate Analysis } \\
\hline & & & & $P$ value & OR & $95 \% \mathrm{Cl}$ \\
\hline \multicolumn{7}{|l|}{ Underlying disease } \\
\hline Hematologic malignancies & $45(13.9)$ & $44(16)$ & I (2) & 0.01 & 15.52 & $1.89-127.14$ \\
\hline Prematurity & $|3|(40.4)$ & $129(46.9)$ & $2(4.1)$ & $<0.01$ & 37.07 & $8.29-165.84$ \\
\hline \multicolumn{7}{|l|}{ Departments } \\
\hline General medical ward & $157(48.5)$ & $119(43.3)$ & $38(77.6)$ & $<0.01$ & 0.2 & $0.08-0.51$ \\
\hline $\mathrm{NICU}$ & $65(20.1)$ & $64(23.3)$ & I (2) & 0.11 & 5.82 & $0.68-49.89$ \\
\hline Previous use invasive mechanical ventilation & $82(25.3)$ & $81(29.5)$ & I (2) & 0.01 & 13.09 & $1.66-103.56$ \\
\hline Antibiotic therapy prior to admission & $90(27.8)$ & $80(29.1)$ & $10(20.4)$ & 0.14 & 2.02 & $0.79-5.19$ \\
\hline
\end{tabular}

Abbreviations: NICU, neonatal intensive care unit; HAI, healthcare-associated infections; CAI, community-acquired infections.

Table 3 Characteristics of the Children with HAl According to Pattern of Carbapenem Resistance (CRKP or CSKP Isolates)

\begin{tabular}{|c|c|c|c|}
\hline Variable & CRKP (N=I|2) & $\operatorname{CSKP}(N=146)$ & $P$ value \\
\hline Age (months) & $0.53(0.12-3.2)$ & $0.65(0.17-12)$ & 0.27 \\
\hline Male & $62(55.4)$ & $82(56.2)$ & 0.9 \\
\hline Rural area & $74(66.1)$ & $72(49.3)$ & $<0.01$ \\
\hline \multicolumn{4}{|l|}{ Underlying disease } \\
\hline Hematologic malignancies & $18(16.1)$ & $26(17.8)$ & 0.71 \\
\hline Congenital anomalies & $19(17)$ & $16(11)$ & 0.16 \\
\hline Prematurity & $58(51.8)$ & $61(41.8)$ & 0.11 \\
\hline NEC & $9(8)$ & $15(10.3)$ & 0.54 \\
\hline Solid tumors & $2(1.8)$ & $\mathrm{I}(0.7)$ & 0.58 \\
\hline Severe malnutrition & $7(6.3)$ & $4(2.7)$ & 0.28 \\
\hline \multicolumn{4}{|l|}{ Departments } \\
\hline - Hematology-oncology ward & $17(15.2)$ & $25(17.1)$ & 0.68 \\
\hline - General medical ward & $56(50)$ & $58(39.7)$ & 0.1 \\
\hline$\bullet \mathrm{NICU}$ & $13(11.6)$ & $44(30.1)$ & $<0.01$ \\
\hline • PICU & $8(7.1)$ & $7(4.8)$ & 0.42 \\
\hline - Surgical ward & $18(16.1)$ & $12(8.2)$ & 0.051 \\
\hline Length of hospitalization & $38.5(24-56.3)$ & $30(19.3-51.8)$ & 0 \\
\hline Length of hospitalization before the onset of KP infections & $12.5(2-24.3)$ & $10(2-22)$ & 0.02 \\
\hline \multicolumn{4}{|l|}{ Invasive procedures and devices } \\
\hline $\mathrm{PICC}$ & $37(33)$ & $43(29.5)$ & 0.54 \\
\hline Thoracic cavity drainage & $6(5.4)$ & $6(4.1)$ & 0.64 \\
\hline Abdominal indwelling catheter & $3(2.7)$ & I (0.7) & 0.32 \\
\hline Indwelling urinary catheter & $\mathrm{I}(0.9)$ & $4(2.7)$ & 0.39 \\
\hline Invasive mechanical ventilation & $51(45.5)$ & $39(26.7)$ & $<0.01$ \\
\hline Surgical operation & $23(20.5)$ & $32(21.9)$ & 0.79 \\
\hline Organ dysfunction (any) & $41(36.6)$ & $22(15.1)$ & $<0.01$ \\
\hline Septic shock & $13(11.6)$ & $13(8.9)$ & 0.48 \\
\hline Mortality or hopeless discharge & $16(14.3)$ & $19(13)$ & 0.77 \\
\hline \multicolumn{4}{|l|}{ Combined organ infection } \\
\hline Pneumonia & $54(48.2)$ & $60(4 I .1)$ & 0.25 \\
\hline Meningitis & $34(30.4)$ & $21(14.4)$ & $<0.01$ \\
\hline Intrabdominal infections & $15(13.4)$ & $23(15.8)$ & 0.6 \\
\hline Upper urinary tract infections & $2(1.8)$ & I (0.7) & 0.58 \\
\hline
\end{tabular}

Abbreviations: CRKP, carbapenem-resistant Klebsiella pneumoniae; CSKP, carbapenem-sensitive Klebsiella pneumoniae; KP, Klebsiella pneumoniae; PICC, peripherally inserted central catheter; NEC, necrotizing enterocolitis; NICU, neonatal intensive care unit; PICU, pediatric intensive care unit. 
Table 4 Multiple Regression Analysis of Risk Factors Associated with CRKP Infections in HAI

\begin{tabular}{|c|c|c|c|c|c|}
\hline \multirow[t]{2}{*}{ Variable } & \multirow[t]{2}{*}{ Total $(\mathbf{N}=\mathbf{2 5 8})$} & \multirow[t]{2}{*}{ CRKP $(\mathrm{N}=|| 2)$} & \multirow[t]{2}{*}{ CSKP $(N=146)$} & \multicolumn{2}{|c|}{ Multi-Variate Analysis } \\
\hline & & & & $P$ value & OR (95\% Cl) \\
\hline Rural area & $146(56.6)$ & $74(66.1)$ & $72(49.3)$ & 0.02 & $1.94(1.12-3.35)$ \\
\hline Congenital anomalies & 35 & $19(17)$ & $16(11)$ & 0.18 & $1.77(0.78-4.01)$ \\
\hline Prematurity & 119 & $58(51.8)$ & $6 I(4 I .8)$ & 0.07 & $1.95(0.95-4.01)$ \\
\hline Surgical prior to admission & $18(7)$ & $8(7.1)$ & $10(6.8)$ & 0.8 & $0.87(0.29-2.57)$ \\
\hline Antibiotic therapy prior to admission & $70(27.1)$ & $42(37.5)$ & $28(19.2)$ & $<0.01$ & $2.33(1.25-4.33)$ \\
\hline Previous use PICC & $73(28.3)$ & $34(30.4)$ & $39(26.7)$ & 0.98 & $0.99(0.46-2.15)$ \\
\hline Previous use invasive mechanical ventilation & $76(29.5)$ & $43(38.4)$ & $33(22.6)$ & 0.02 & $2.08(1.12-3.88)$ \\
\hline Hospital stay prior to admission in 30 days & $101(39.1)$ & $57(50.9)$ & $44(30.1)$ & $<0.01$ & $3.46(|.87-6.4|)$ \\
\hline
\end{tabular}

Abbreviation: PICC, peripherally inserted central catheter.

Table 5 Multiple Regression Analysis of Risk Factors Associated with Poor Outcomes in HAI

\begin{tabular}{|c|c|c|c|c|c|c|}
\hline \multirow[t]{2}{*}{ Variable } & \multirow{2}{*}{$\begin{array}{l}\text { Survived } \\
\mathrm{N}=236\end{array}$} & \multirow{2}{*}{$\begin{array}{l}\text { Poor Outcome } \\
N=39\end{array}$} & \multicolumn{2}{|c|}{ Univariate Analysis } & \multicolumn{2}{|c|}{ Multi-Variate Analysis } \\
\hline & & & $P$ value & OR (95\% Cl) & $P$ value & OR $(95 \% \mathrm{CI})$ \\
\hline Age (months) & $0.6(0.1-4.8)$ & $1.0(0.4-6.7)$ & 0.75 & $1.0(0.99-1.0)$ & & \\
\hline Male & $132(55.9)$ & $22(56.4)$ & 0.96 & $1.02(0.52-2.02)$ & & \\
\hline Rural area & $132(55.9)$ & $24(61.5)$ & 0.51 & $1.26(0.63-2.52)$ & & \\
\hline \multicolumn{7}{|l|}{ Underlying disease } \\
\hline Hematologic malignancies & $39(16.5)$ & $5(12.8)$ & 0.56 & $0.74(0.27-2.02)$ & & \\
\hline Congenital anomalies & $31(13.1)$ & $6(15.4)$ & 0.7 & $1.2(0.47-3.1)$ & & \\
\hline NEC & $22(9.3)$ & $5(12.8)$ & 0.5 & $\mathrm{I} .43(0.5 \mathrm{I}-4.03)$ & & \\
\hline Prematurity & $3(1.3)$ & I (2.6) & 0.92 & $0.97(0.49-1.9)$ & & \\
\hline Solid tumors & $3(1.3)$ & I (2.6) & 0.54 & $2.04(0.2 \mathrm{I}-20.16)$ & & \\
\hline Severe malnutrition & $9(3.8)$ & $2(5.1)$ & 0.7 & $1.36(0.28-6.56)$ & & \\
\hline Immunodeficiencies & $2(0.8)$ & I (2.6) & 0.36 & $3.08(0.27-34.79)$ & & \\
\hline $\begin{array}{l}\text { Length of hospitalization before the } \\
\text { onset of BSI }\end{array}$ & $10(2-12)$ & $10(2-11)$ & 0.44 & $0.99(0.97-1.01)$ & & \\
\hline \multicolumn{7}{|l|}{ Invasive procedures and devices } \\
\hline PICC & $69(29.2)$ & $13(33.3)$ & 0.61 & $1.21(0.59-2.49)$ & & \\
\hline Thoracic cavity drainage & II (4.7) & I (2.6) & 0.56 & $0.54(0.07-4.29)$ & & \\
\hline Abdominal indwelling catheter & $3(1.3)$ & $\mathrm{I}(2.6)$ & 0.54 & $2.04(0.21-20.16)$ & & \\
\hline Indwelling urinary catheter & $4(1.7)$ & I (2.6) & $0.7 \mathrm{I}$ & $1.53(0.17-14.03)$ & & \\
\hline Invasive mechanical ventilation & $77(32.6)$ & $19(48.7)$ & 0.05 & $1.96(0.99-3.89)$ & 0.88 & $0.93(0.39-2.21)$ \\
\hline Operation & $5 I(2 \mid .6)$ & $9(23.1)$ & 0.84 & $1.09(0.49-2.44)$ & & \\
\hline Organ dysfunction (any) & $50(2 \mid .2)$ & $19(48.7)$ & 0 & $3.53(1.75-7.13)$ & 0.02 & $2.92(1.23-6.95)$ \\
\hline Septic shock & $21(8.9)$ & $9(23.1)$ & 0.01 & $3.07(1.29-7.33)$ & 0.08 & $2.29(0.9-5.85)$ \\
\hline Isolation of CRKP $(\mathrm{N}=304)$ & $96(35.7)$ & $16(45.7)$ & 0.25 & & & \\
\hline \multicolumn{7}{|l|}{ Combined organ infection } \\
\hline Pneumonia & $98(4 \mid .5)$ & $21(53.8)$ & 0.15 & $1.64(0.83-3.25)$ & 0.58 & $1.22(0.59-2.53)$ \\
\hline Meningitis & $53(22.5)$ & II (28.2) & 0.43 & $1.36(0.63-2.91)$ & 0.31 & $0.52(0.15-1.85)$ \\
\hline Intrabdominal infections & $38(16.1)$ & $3(7.7)$ & 0.18 & $0.43(0.13-1.48)$ & & \\
\hline Upper urinary tract infections & $3(1.3)$ & $0(0)$ & - & - & & \\
\hline
\end{tabular}

Abbreviations: $\mathrm{HAI}$, healthcare-associated infections; PICC, peripherally inserted central catheter; NEC, necrotizing enterocolitis; NICU, neonatal intensive care unit; $\mathrm{PICU}$, pediatric intensive care unit; CRKP, carbapenem-resistant Klebsiella pneumoniae. 
Table 6 Resistance Patterns of Klebsiella pneumoniae

\begin{tabular}{|c|c|c|c|c|}
\hline \multirow[t]{2}{*}{ Antimicrobial Drugs } & \multicolumn{4}{|c|}{ Percentage Rate (\%) of Resistance ${ }^{a}$ (Number of Isolates Resistant/Number of Isolates Tested) } \\
\hline & Total & HAI & CAI & $P$ value \\
\hline Ampicillin & $93.8(227 / 242)$ & $96.6(199 / 206)$ & $77.8(28 / 36)$ & 0 \\
\hline Ampicillin/Sulbactam & $75.6(205 / 27 I)$ & $81.5(190 / 233)$ & $39.5(15 / 38)$ & 0 \\
\hline Cefepime & $55.3(167 / 302)$ & $60.5(155 / 256)$ & $26.1(12 / 46)$ & 0 \\
\hline Ceftriaxone & $59.8(76 / 127)$ & $70.2(73 / 104)$ & $13(3 / 23)$ & 0 \\
\hline Ceftazidime & $62.7(188 / 300)$ & $69.4(177 / 255)$ & $24.4(1 \mathrm{I} / 45)$ & 0 \\
\hline Cefuroxime & $58.6(17 / 29)$ & $66.7(16 / 24)$ & $20(1 / 5)$ & 0.13 \\
\hline Piperacillin/tazobactam & $43(128 / 298)$ & $48.6(124 / 255)$ & $9.3(4 / 43)$ & 0 \\
\hline Gentamicin & $30.6(92 / 301)$ & $34.5(88 / 255)$ & $8.7(4 / 46)$ & 0 \\
\hline Amikacin & I4.2 (43/302) & $16.4(42 / 256)$ & $2.2(1 / 46)$ & 0.02 \\
\hline Ciprofloxacin & $20.8(63 / 303)$ & $23.7(61 / 257)$ & $4.3(2 / 46)$ & $<0.01$ \\
\hline Levofloxacin & I8.2 (55/303) & $21(54 / 257)$ & $2.2(1 / 46)$ & $<0.01$ \\
\hline Imipenem & $36.2(110 / 304)$ & $41.5(107 / 258)$ & $6.5(3 / 46)$ & 0 \\
\hline Meropenem & $40.5(96 / 237)$ & $45.4(93 / 205)$ & $9.4(3 / 32)$ & 0 \\
\hline Tigecycline & $10.5(2 / 19)$ & $12.5(2 / 16)$ & $0(0 / 3)$ & 1 \\
\hline Polymycin B & $0.6(1 / 169)$ & $0.7(1 / 148)$ & $0(0 / 2 I)$ & 1 \\
\hline
\end{tabular}

Note: ${ }^{a}$ Klebsiella pneumoniae isolates displaying intermediate susceptibility were not categorized as resistance in this study.

Abbreviations: HAl, healthcare-associated infections; CAl, community-acquired infections.

\section{Discussion}

This multicenter retrospective study first revealed that most episodes of invasive KP infections were healthcareassociated in pediatric patients in China. We further demonstrated that the high percentage of CRKP infections usually occurred in healthcare setting (40.4\%) rather than in community setting (10.2\%). Of particularly concern, $58.9 \%$ of pediatric invasive healthcare-associated KP infections occurred in neonates. KP infections impose a health threat to hospitalized neonates and children.

According to the surveillance in Chinese adults, KP was the second common pathogen in hospital-associated bloodstream infections. ${ }^{16}$ Our recent study revealed that KP was the second common pathogen of bloodstream bacterial infections in Chinese pediatric patients. ${ }^{4}$ Our present study further showed that $84.9 \%$ of invasive KP infections were hospital-associated. Thus, it is feasible to prevent and reduce invasive KP infections through a bundle of effective infection prevention and control (IPC) measures. Identifying some key risk factors is essential to formulate the targeted measures. In this study, hematologic malignancies, prematurity, and invasive mechanical ventilation were identified as the independent risk factors for HAI. The available evidences have demonstrated that IPC practices and procedures can effectively prevent the occurrence and the spread of HAI in healthcare facilities. ${ }^{17,18}$ Thus, reinforcement of IPC at high-risk pediatric units, such as hematological units, pediatric and neonatal intensive care units, is key to prevent invasive healthcare-associated KP infections. Healthcare-associated $\mathrm{KP}$ infections in preterm neonates are of particular concern because $58.9 \%$ of invasive healthcare-associated KP occurred in neonate and the odds ratio for prematurity is highest. Prematurity is a common risk factor of any nosocomial infections in neonatal units. ${ }^{19,20}$ Our results further support this finding and emphasize prematurity as the most key risk factor of severe invasive nosocomial KP infections. Therefore, hospitalized preterm neonates should be placed on the highest priority group for whom IPC practice should be strengthened and improved. Besides, we noticed that $43.3 \%$ of children with hospital-associated KP infections had accompanying pneumonia and 34.9\% of patients received mechanical ventilation therapy. Thus, part episodes of invasive healthcare-associated KP infections were likely to be secondary to ventilation-associated pneumonia.

We noticed that the high prevalence of CRKP in hospital setting but low prevalence of CRKP in community setting in pediatric patients. CRKP indeed represents a clinical and economic impact on pediatric patients and tertiary pediatric hospital in China. In this study, previous antibiotic therapy prior to hospitalization, previous mechanical ventilation, and prior hospital stay within the past 30 days were independent risk factors for acquisition of healthcare-associated CRKP infections, which is 
consistent with most of previous studies conducted in children and adults. ${ }^{21-23}$ In addition, we found that children from rural area were an independent risk factor for invasive healthcare-associated CRKP infections in Chinese children. In China, it is usual for rural patients to delay seeking medical consultation, furthermore, severe rural patients usually cannot have access to the tertiary hospitals for initial treatment, and they have to experience the hospital referral for advanced treatment. Inappropriate treatment is unavoidable before referral to the tertiary hospitals. Thus, rural children are at higher risk of acquiring HAI. Unlike most previous single-center or specificunit studies, ${ }^{23,24}$ we did not find that patients with hematologic malignancies were an independent risk factor of invasive CRKP infections. This difference is possibly owing to the selected bias of study hospitals and enrolled patients.

Although a few of studies suggested HAI was a risk factor for mortality of KP infections based on univariate analyses, $^{25-27}$ we found no statistical difference in clinical outcomes of invasive KP infections between HAI and CAI patients. This discrepancy is probably owing to loss to follow-up in discharged patients in this cohort. However, HAI patients were more likely to develop organ dysfunction, which was the only independent risk factor of poor outcomes. Most episodes (79.3\%) of invasive healthcareassociated KP infections are accompanied by focal organ involvement, of which, pneumonia (43.3\%) and meningitis $(23.3 \%)$ were the common manifestations. Of note, KPassociated meningitis almost occurred in HAI patients. HAI patients usually had underlying diseases or were immunocompromised, thus, those patients were more likely to develop meningitis secondary to bloodstream infection or primary site infection, especially premature infants. These findings add our understanding of clinical presentation of invasive healthcare-associated KP infections, which will be helpful to predict the possible source and secondary complications of invasive KP infections and select appropriate antibiotic regimen. In African countries, the mortality rate of bloodstream KP infections in pediatric patients was reported to reach as high as $21.6 \%-56.5 \%,{ }^{8,11,28}$ significantly higher than our rough estimation $(13.6 \%)$ in Chinese children. This could reflect the regional difference in accessibility of tertiary pediatric medical resource and capacity of pediatric critical intensive care for pediatric patients. On the other hand, virulence factors of endemic KP isolates are also a contributing factor of the fatal outcomes. ${ }^{29,30}$ We need to further characterize the virulence profile of KP strains in the next step to understand KP pathogenicity well.

Of particular concern is that healthcare-associated KP strains displayed higher prevalence of resistance to the tested antibiotics than community-acquired KP isolates, especially to extended-spectrum cephalosporin and carbapenem. The frequency of ceftriaxone-resistant healthcare-associated KP and community-acquired KP was $70.2 \%$ and $13.0 \%$, respectively, and the frequency of meropenem-resistant healthcareassociated KP and meropenem-resistant community-acquired KP was $45.4 \%$ and $9.4 \%$, respectively. The high prevalence of $\mathrm{KP}$ resistance in hospital setting makes it difficult to empirically and definitely select antibiotic therapy for invasive healthcare-associated KP infections in pediatric patients because the prescription of polymyxin $\mathrm{B}$ and tigecycline, which are almost sensitive to KP, are strictly restricted to use in children due to safety consideration, especially in neonates. $^{31}$

Our study first described important profiles of healthcare-associated KP infections in Chinese pediatric patients and adds some knowledge and understanding of invasive KP and CRKP infections in children. Although this study is a retrospective study, the enrolled patients from all pediatric units of the nine tertiary children's hospitals are representative, and all episodes of invasive $\mathrm{KP}$ infections are culture-proven. However, our study has two major limitations. Firstly, retrospective data collection probably resulted in clinical information missing, such as prior healthcare history, prior previous antibiotic therapy and PICC utilization. Second, there is a potential estimation bias of clinical outcomes because KPassociated death post discharge was not captured. Further prospective studies are needed to trace the potential transmission source of $\mathrm{KP}$ in hospital setting and explain the disease severity based on integration clinical data with molecular mechanisms of carbapenemases and virulence factors of KP strains. The efforts will help to take intensified and effective IPC measures to reduce healthcare-associated KP infections and CRKP infections in pediatric patients.

\section{Conclusion}

Pediatric invasive KP infections and high prevalence of CRKP infections largely occurred in healthcare settings in China. Of particular note, we found prematurity is the most key risk factor for healthcare-associated KP infections, and children from rural area were also an independent risk factor for CRKP infections. Overall, organ 
dysfunction is independently correlated with poor outcomes. The adequate and intensified infection control measures should be focused on high-risk hematologic patients, premature neonatal patients and intubated patients.

\section{Ethical Approval}

This study was reviewed and approved by the Ethics Committee of Fudan Children's Hospital (ethics approval number: 2020471). Informed consent from patients was not required by the Ethics Committee, because there was no contact with patients and all data were deidentified. This study was conducted in accordance with the Declaration of Helsinki.

\section{Acknowledgment}

We thank Prof Hong Zhao, the secretary of China Society of Infectious Diseases, for her help with the program initiation. We thank the members of the collaborative working group of pediatric subgroups of China Society of Infectious Diseases for their collecting the data for this study.

\section{Funding}

This research did not receive any specific grant from funding agencies in the public, commercial, or not-forprofit sectors.

\section{Disclosure}

All authors declared no potential conflicts of interest to disclose.

\section{References}

1. Podschun R, Ullmann U. Klebsiella spp. as nosocomial pathogens: epidemiology, taxonomy, typing methods, and pathogenicity factors. Clin Microbiol Rev. 1998;11(4):589-603. doi:10.1128/CMR.1 1.4.589

2. Diekema DJ, Hsueh PR, Mendes RE, et al. The microbiology of bloodstream infection: 20-year trends from the SENTRY antimicrobial surveillance program. Antimicrob Agents Chemother. 2019;63(7). doi:10.1128/AAC.00355-19

3. Bielicki JA, Lundin R, Sharland M. Antibiotic resistance prevalence in routine bloodstream isolates from children's hospitals varies substantially from adult surveillance data in Europe. Pediatr Infect Dis $J$. 2015;34(7):734-741. doi:10.1097/INF.0000000000000652

4. Qiu Y, Yang J, Chen Y, et al. Microbiological profiles and antimicrobial resistance patterns of pediatric bloodstream pathogens in China, 2016-2018. Eur J Clin Microbiol Infect Dis. 2021;40(4):739-749. doi:10.1007/s10096-020-04069-2

5. Nordmann P, Cuzon G, Naas T. The real threat of Klebsiella pneumoniae carbapenemase-producing bacteria. Lancet Infect Dis. 2009;9 (4):228-236. doi:10.1016/S1473-3099(09)70054-4

6. Tacconelli E, Carrara E, Savoldi A, et al. Discovery, research, and development of new antibiotics: the WHO priority list of antibiotic-resistant bacteria and tuberculosis. Lancet Infect Dis. 2018;18(3):318-327. doi:10.1016/S1473-3099(17)30753-3
7. Hu F, Guo Y, Yang Y, et al. Resistance reported from China antimicrobial surveillance network (CHINET) in 2018. Eur J Clin Microbiol Infect Dis. 2019;38(12):2275-2281. doi:10.1007/s10096019-03673-1

8. Ballot DE, Bandini R, Nana T, et al. A review of -multidrug-resistant Enterobacteriaceae in a neonatal unit in Johannesburg, South Africa. BMC Pediatr. 2019;19(1):320.

9. Pokhrel B, Koirala T, Shah G, Joshi S, Baral P. Bacteriological profile and antibiotic susceptibility of neonatal sepsis in neonatal intensive care unit of a tertiary hospital in Nepal. BMC Pediatr. 2018;18 (1):208. doi:10.1186/s12887-018-1176-x

10. Nabarro LEB, Shankar C, Pragasam AK, et al. Clinical and bacterial risk factors for mortality in children with carbapenem-resistant Enterobacteriaceae bloodstream infections in India. Pediatr Infect Dis J. 2017;36(6):e161-e6. doi:10.1097/INF.0000000000001499

11. Buys H, Muloiwa R, Bamford C, Eley B. Klebsiella pneumoniae bloodstream infections at a South African children's hospital 20062011, a Cross-Sectional Study. BMC Infect Dis. 2016;16(1):570. doi:10.1186/s12879-016-1919-y

12. Lake JG, Weiner LM, Milstone AM, Saiman L, Magill SS, See I. Pathogen distribution and antimicrobial resistance among pediatric healthcare-associated infections reported to the national healthcare safety network, 2011-2014. Infect Control Hosp Epidemiol. 2018;39 (1):1-11. doi:10.1017/ice.2017.236

13. Russotto V, Cortegiani A, Fasciana T, et al. What healthcare workers should know about environmental bacterial contamination in the intensive care unit. Biomed Res Int. 2017;2017:6905450. doi:10.1155/2017/6905450

14. Guh AY, Bulens SN, Mu Y, et al. Epidemiology of carbapenemresistant enterobacteriaceae in 7 US communities, 2012-2013. JAMA. 2015;314(14):1479-1487. doi:10.1001/jama.2015.12480

15. Clinical and Laboratory Standards Institute (CLSI). Performance Standards for Antimicrobial Susceptibility Testing. M100. 28th ed. Wayne, PA; 2018.

16. Li SG, Liao K, Su DH, et al. [Analysis of pathogen spectrum and antimicrobial resistance of pathogens associated with hospital-acquired infections collected from 11 teaching hospitals in 2018]. Zhonghua Yi Xue Za Zhi. 2020;100(47):3775-3783. Chinese. doi:10.3760/cma.j.cn112137-20200430-01389

17. WHO Guidelines Approved by the Guidelines Review Committee. Guidelines for the Prevention and Control of CarbapenemResistant Enterobacteriaceae, Acinetobacter Baumannii and Pseudomonas Aeruginosa in Health Care Facilities. Geneva: World Health Organization, Copyright (C) World Health Organization 2017; 2017.

18. Murni IK, Duke T, Kinney S, Daley AJ, Soenarto Y. Reducing hospital-acquired infections and improving the rational use of antibiotics in a developing country: an effectiveness study. Arch Dis Child. 2015;100(5):454-459. doi:10.1136/archdischild-2014-307297

19. Rettedal S, Høyland Löhr I, Natås O, Sundsfjord A, Øymar K. Risk factors for acquisition of CTX-M-15 extended-spectrum beta-lactamase-producing Klebsiella pneumoniae during an outbreak in a neonatal intensive care unit in Norway. Scand $J$ Infect Dis. 2013;45(1):54-58. doi:10.3109/00365548.2012.713116

20. Giannoni E, Agyeman PKA, Stocker M, et al. Neonatal sepsis of early onset, and hospital-acquired and community-acquired late onset: a Prospective Population-Based Cohort Study. J Pediatr. 2018;201:106-14.e4. doi:10.1016/j.jpeds.2018.05.048

21. Gómez Rueda V, Zuleta Tobón JJ. Risk factors for infection with carbapenem-resistant Klebsiella pneumoniae: a case-case-control study. Colomb Med. 2014;45(2):54-60. doi:10.25100/cm.v45i2.1417

22. Hussein K, Raz-Pasteur A, Finkelstein R, et al. Impact of carbapenem resistance on the outcome of patients' hospital-acquired bacteraemia caused by Klebsiella pneumoniae. $J$ Hosp Infect. 2013;83 (4):307-313. doi:10.1016/j.jhin.2012.10.012 
23. Akturk H, Sutcu M, Somer A, et al. Carbapenem-resistant Klebsiella pneumoniae colonization in pediatric and neonatal intensive care units: risk factors for progression to infection. Braz J Infect Dis. 2016;20(2):134-140. doi:10.1016/j.bjid.2015.12.004

24. Zhang Y, Guo LY, Song WQ, Wang Y, Dong F, Liu G. Risk factors for carbapenem-resistant $\mathrm{K}$. pneumoniae bloodstream infection and predictors of mortality in Chinese paediatric patients. BMC Infect Dis. 2018;18(1):248. doi:10.1186/s12879-018-3160-3

25. Folgori L, Livadiotti S, Carletti M, et al. Epidemiology and clinical outcomes of multidrug-resistant, gram-negative bloodstream infections in a European tertiary pediatric hospital during a 12-month period. Pediatr Infect Dis J. 2014;33(9):929-932. doi:10.1097/ INF.0000000000000339

26. Jung Y, Lee MJ, Sin HY, et al. Differences in characteristics between healthcare-associated and community-acquired infection in community-onset Klebsiella pneumoniae bloodstream infection in Korea. BMC Infect Dis. 2012;12:239. doi:10.1186/1471-2334-12-239

27. Wu HS, Wang FD, Tseng $\mathrm{CP}$, Wu TH, Lin YT, Fung CP. Characteristics of healthcare-associated and community-acquired Klebsiella pneumoniae bacteremia in Taiwan. J Infect. 2012;64 (2):162-168. doi:10.1016/j.jinf.2011.11.005
28. Pereira CA, Marra AR, Camargo LF, et al. Nosocomial bloodstream infections in Brazilian pediatric patients: microbiology, epidemiology, and clinical features. PLoS One. 2013;8(7):e68144. doi:10.1371/ journal.pone.0068144

29. Gu D, Dong N, Zheng Z, et al. A fatal outbreak of ST11 carbapenem-resistant hypervirulent Klebsiella pneumoniae in a Chinese hospital: a molecular epidemiological study. Lancet Infect Dis. 2018;18(1):37-46. doi:10.1016/S1473-3099(17)30489-9

30. Fasciana T, Gentile B, Aquilina M, et al. Co-existence of virulence factors and antibiotic resistance in new Klebsiella pneumoniae clones emerging in south of Italy. BMC Infect Dis. 2019;19(1):928. doi:10.1186/s12879-019-4565-3

31. European Medicines Agency. Available from: https://www.ema. europa.eu/en/documents/product-information/tygacil-epar-productinformation_en.pdf. Accessed June 28, 2021.

\section{Publish your work in this journal}

Infection and Drug Resistance is an international, peer-reviewed openaccess journal that focuses on the optimal treatment of infection (bacterial, fungal and viral) and the development and institution of preventive strategies to minimize the development and spread of resistance. The journal is specifically concerned with the epidemiology of antibiotic resistance and the mechanisms of resistance development and diffusion in both hospitals and the community. The manuscript management system is completely online and includes a very quick and fair peerreview system, which is all easy to use. Visit http://www.dovepress.com/ testimonials.php to read real quotes from published authors. 